\title{
EFEITO DO EXERCÍCIO COM 1 E 3 MINUTOS DE INTERVALO DE DESCANSO ENTRE AS SÉRIES NA ATIVIDADE SÉRICA DAS TRANSAMINASES
}

\author{
Ramon Martins Alves ${ }^{1}$; Roberta Sobreira ${ }^{1}$; Joelen Soares de Castro ${ }^{1}$; Paulo Vinícios Zovico ${ }^{1}$; \\ Wilkes de Oliveira $^{2} \&$ Victor Magalhães Curty ${ }^{3 *}$
}

http://dx.doi.org/10.18571/acbm.007

1 - Universidade Iguaçu Campus V, Itaperuna, RJ, Brasil

2 - Laboratorio de Analises Clínicas Kashima, Guaçuí, ES, Brasil.

3 - Faculdade Santo Antonio de Pádua - FASAP, Santo Antônio de Pádua, RJ, Brasil.

*Autor para correspondência: victorcurty_personal@hotmail.com

\section{RESUMO}

O treinamento de força (TF) intenso ocasiona danos na estrutura muscular, que podem ser detectados por meio de marcadores indiretos. OBJETIVO. Verificar se há alterações na atividade da creatina quinase (CK), da aspartato transaminase (AST) e alanina transaminase (ALT) com o TF em diferentes intervalos entre séries e exercícios. MÉTODOS. Treze voluntários ( $\pm 21,8$ anos; $\pm 173,6 \mathrm{~cm} ; \pm 68,3 \mathrm{~kg}$ ) realizaram o teste de uma repetição máxima (1RM) nos exercícios: supino reto, puxada (pulley), desenvolvimento sentado, tríceps (pulley) e rosca bíceps. Após sete dias, todos foram submetidos a uma coleta de sangue (PRE) e realizaram 4 séries máximas até a falha concêntrica com intensidade de $85 \%$ de $1 \mathrm{RM}$ e intervalos de 1 ou 3 minutos entre as séries e exercícios. Todos retornaram ao mesmo local 24, 48 e 72 horas após a sessão para mais uma coleta de sangue. Repetindo os procedimentos após uma semana, porém os voluntários que realizaram o teste com intervalo de 1 minuto na semana seguinte manteriam intervalos de 3 minutos e vice-versa. Realizou-se ANOVA mantendo como significância $\mathrm{p}<0,05$. RESULTADOS. Houve redução significativa do número de repetições a cada série de todos os exercícios com 1 e 3 minutos de intervalo. Observou-se um aumento significativo da atividade sérica de CK e de AST 24, 48 e 72 horas utilizando ambos os intervalos. ALT não se alterou. CONCLUSÃO. O TF causou aumento na atividade de CK e AST, mas sem apresentar diferenças com os intervalos utilizados. Porém, pequenos intervalos reduzem o volume total de repetições.

Palavras-chave: treinamento resistido, músculo esquelético; força; creatina-quinase; fadiga.

\begin{abstract}
The heavy resistance strength training causes damage to the muscle structure that can be detected through indirect markers. OBJECTIVE. Examine whether there are changes in creatine kinase (CK), aspartate transaminase (AST) and alanine transaminase (ALT) activity with the resistance strength training in different intervals between sets and exercises. METHODS. Thirteen volunteers $( \pm 21,8$ years; $\pm 173,6 \mathrm{~cm} ; \pm 68,3 \mathrm{Kg}$ ) performed the one repetition maximum test (1RM) in the exercises: bench press, driven (pulley), sitting development, tríceps (pulley) and biceps. After seven days all were submitted a blood sample (PRE) and they performed 4 maximum sets by concentric failure with intensity the $85 \%$ of $1 \mathrm{RM}$ and intervals 1 and 3 minutes between sets and exercises. All returned to the same place
\end{abstract}


24, 48 and 72 hours after the after the session to another blood sample. Repeating the procedures after one week, but the volunteers who performed the test with an interval of 1 minute next week, they would keep the intervals of 3 minutes and vice versa. ANOVA was performed keeping as significant $p<0,05$. RESULTS. Was a significant reduction the number of repetitions in each set of all exercises with 1 and 3 minutes apart. There was a significant increase activity of serum CK and AST 24, 48 and 72 hours using both ranges. ALT did not change. CONCLUSION. The heavy resistance strength training caused increased activity of CK and AST but no differences with the intervals used. However, short intervals reduce the total volume of repeats.

Keywords: Tesistance training, skeletal muscle, strength, creatine kinase, fatigue.

\section{1 - Introdução}

O treinamento de força (TF) é eficiente para aumentar força, hipertrofia, potência e resistência muscular, além de melhorar a qualidade de vida (BARROSO et al., 2005; FOSCHINI et al., 2007; SIMÃO et al., 2008). Nos últimos anos tem crescido o número de praticantes que desejam suprir suas necessidades, sejam elas estéticas, terapêuticas, melhoria da qualidade de vida e etc. Devido essa importância atribuída ao treinamento de força, muitos estudos (SIMÃO et al., 2006a; SIMÃO et al., 2006b; FOSCHINI et al., 2009; PRESTES et al., 2010; SILVA et al., 2011) têm sido feitos para compreender o que envolve as variáveis deste treinamento.

Entre as variáveis fundamentais do treinamento (por ex.: volume, intensidade, ordem dos exercícios, velocidade de execução, etc.) tem se destacado nos últimos anos o estudo das respostas fisiológicas quando diferentes tempos de intervalos para descanso entre as séries são proporcionados (LIMA et al., 2006; SALLES et al., 2006; SIMÃO et al., 2006a; SIMÃO et al., 2006b; MIRZAEI et al., 2008; SIMÃO et al., 2008). Essa variável torna-se importante por modular de forma crítica o volume e a intensidade do treino. Tem sido demonstrado que a redução do tempo entre as séries reduz o volume total de treinamento pela redução do número de repetições (LIMA et al., 2006; SALLES et al., 2006; SIMÃO et al., 2006a; SIMÃO et al., 2006b; SIMÃO et al., 2008; MIRANDA et al., 2007; MIRZAEI et al., 2008; SENNA et al., 2009; RODRIGUES et al., 2010b). Contudo exercícios realizados com intervalos curtos (i.e, menores que 60 segundos) aumentam a produção e secreção de hormônios anabólicos e fatores de crescimento (WILLARDSON, 2006; PRESTES et al., 2010).

A lesão muscular é definida como uma desestruturação das fibras musculares diversas vezes decorrentes da prática de atividades físicas, e é comum que elas ocorram em pequena dimensão caracterizando uma resposta plástica importante do músculo esquelético a atividade realizada. As micro-lesões disparam um processo para reestruturar as fibras através de uma resposta inflamatória e da formação e liberação de hormônios e de fatores de crescimento (TRICOLI, 2001; MACHADO, 2007; FOSCHINI et al., 2007; MACHADO, 2008). Em função disso alguns estudos procuraram investigar os efeitos de um exercício (MAYHEW et al, 2005; EVANGELISTA et al., 2011) ou de uma sequência de exercícios (RIBEIRO et al., 2008; RODRIGUES et al., 2010a; MACHADO \& WILLARDSON, 2010; MACHADO et al., 2010; MACHADO et al., 2011) nos marcadores de lesão muscular. Exceto um estudo (MAYHEW et al., 2005) que verificou maior atividade sérica de Creatina quinase (CK) no grupo que realizou as séries com intervalos mais curtos, todos os outros não verificaram 
diferenças significativas (EVANGELISTA et al., 2011; RIBEIRO et al., 2008; RODRIGUES et al., 2010a; MACHADO \& WILLARDSON, 2010; MACHADO et al., 2011).

Danos musculares podem ser detectados indiretamente na corrente sanguinea através da dosagem de enzimas como a $\mathrm{CK}$, lactato desidrogenase (LDH), aspartato aminotranferase (AST) e alanina aminotranferase (ALT) (MOTTA, 2003; FOSCHINI et al., 2007, MACHADO et al., 2010), contudo o uso das transaminases (AST e ALT) tem sido menos freqüente, possivelmente pela menor especificidade (MOTTA, 2003). Desta forma, o objetivo do presente estudo foi verificar se há alterações na atividade sérica das transaminases AST e ALT e da CK induzidas por uma sequência de exercícios de força. Além disso, verificar se diferentes intervalos de descanso entre as séries determinam respostas diferentes desses marcadores indiretos do dano muscular.

\section{2 - Material e Métodos}

\section{1 - Amostra}

Treze voluntários (tabela 1), que exercem atividades físicas monitoradas, do sexo masculino, saudáveis e não usuários de suplementos nutricionais participaram do presente estudo. Todos assinaram termo de participação consentida conforme a resolução $\mathrm{n}^{\mathbf{0}} 251$, de 07/08/1997 do CONSELHO NACIONAL DE SAÚDE e a resolução n ${ }^{\circ}$ 196, de 10/10/1996 que são as diretrizes e normas regulamentadoras de pesquisa envolvendo seres humanos.

Tabela 1 - Características dos Sujeitos

\begin{tabular}{llll}
\hline & Média & Desvio Padrão & Amplitude \\
\hline Idade (anos) & 21,8 & $\pm 2,4$ & $18-26$ \\
Estatura $(\mathrm{cm})$ & 173,6 & $\pm 5,7$ & $163-183$ \\
Massa Corporal $(\mathrm{Kg})$ & 68,3 & $\pm 4,4$ & $60,7-74,4$
\end{tabular}

\section{2 - Protocolo de Teste}

No primeiro dia os sujeitos foram avaliados antropometricamente (perimetria, dobras cutâneas, estatura e massa corporal total) e realizaram teste de uma repetição máxima (1RM) dos exercícios supino reto, puxada no pulley, desenvolvimento sentado, tríceps no pulley e rosca bíceps. Depois do intervalo de 3 dias foi realizado um segundo teste de 1RM. Os testes de $1 \mathrm{RM}$ foram realizados sempre na seqüência descrita.

Sete dias depois os indivíduos realizaram uma coleta de sangue (PRE). Após esta coleta, os participantes foram submetidos a uma sessão com os mesmos exercícios, sendo que para cada exercício foram realizadas 4 séries máximas (até exaustão voluntária) a $85 \%$ de 1RM. De forma contrabalanceada os indivíduos realizaram os exercícios com intervalo de repouso de 1 ou 3 minutos entre séries e exercícios. Vinte e quatro horas, quarenta e oito horas e setenta e duas horas após o exercício os sujeitos retornavam ao local de coleta de sangue para nova coleta.

Uma semana depois os participantes retornaram ao local dos testes e estes realizaram os mesmo procedimentos, porém os voluntários que na primeira semana realizaram os exercícios propostos no teste com intervalos de 1 minuto entre as séries e os exercícios 
realizariam os exercícios na semana seguinte com intervalos de 3 minutos. $\mathrm{O}$ mesmo procedimento seria feito com os participantes que realizaram os exercícios com intervalos de 3 minutos na primeira semana, estes se submeteram aos exercícios com 1 minuto de intervalo entre as séries e exercícios na segunda semana.

\section{3 - Protocolo de Coleta de Sangue e Dosagens}

Aproximadamente $5 \mathrm{ml}$ de sangue venoso foram coletados do antebraço de cada participante. Esta alíquota foi colocada em tubos para ser centrifugada, a $3000 \mathrm{rpm}(1600 \mathrm{G})$ por 20 minutos, e teve o soro separado para dosagens das enzimas Creatina quinase (CK), alanina-transaminase (ALT) e aspartato-transaminase (AST). A coleta foi realizada por profissional habilitado vinculado ao laboratorio de analises clínicas Kashima do município de Guaçuí, ES, Brasil, nas condições de higiene exigidas pelos órgãos públicos responsáveis. Para dosagem das enzimas foi utilizado o método espectrofotométrico a 340 nanômetros $(\mathrm{nm})$ através do ensaio cinético ultravioleta. $\mathrm{O}$ aparelho utilizado é o espectrofotômetro da marca Bio Plus e o kit utilizado foi o LabTest.

\section{4 - Análise Estatística}

Os resultados foram analisados estatisticamente utilizando o teste de KolmogorovSmirnov, resultando que a distribuição dos dados era gaussiana (curva normal), possibilitando assim a utilização da Analise de Variância (ANOVA) para avaliar a hipótese nula de que as médias das variáveis de cada grupo eram iguais, versus a hipótese alternativa de que as médias eram diferentes. Foi utilizado o teste de Sheffée como post hoc quando necessário. A potência dos testes foi fixada em $5 \%(\mathrm{p}<0,05)$. O tratamento estatístico foi realizado em SPSS ${ }^{\circledR} 13.0$ for Windows (LEAD Technologies, 2004).

\section{3 - Resultados}

A tabela 2 mostra os resultados médios do teste de 1RM.

Tabela 2 - Resultados do teste de 1RM.

\begin{tabular}{lcc}
\hline Exercícios & $1^{\circ}$ teste & $2^{\circ}$ teste \\
\hline Supino Reto $(\mathrm{Kg})$ & $78,1 \pm 13,9$ & $81,4 \pm 12,8$ \\
Puxada no Pulley $(\mathrm{Kg})$ & $91,8 \pm 6,9$ & $95,5 \pm 8,6$ \\
Desenvolvimento $(\mathrm{Kg})$ & $68,0 \pm 11,6$ & $71,8 \pm 11,4$ \\
Rosca Tríceps $(\mathrm{Kg})$ & $82,5 \pm 11,6$ & $88,4 \pm 14,1$ \\
Rosca Bíceps $(\mathrm{Kg})$ & $48,0 \pm 8,0$ & $50,0 \pm 7,9$ \\
\hline
\end{tabular}

O gráfico 1 mostra o número de repetições por série de cada um dos exercícios executados com intervalos de descanso de 1 e 3 minutos. Houve uma redução significativa do número de repetições a cada série executada e uma diferença entre os grupos nas séries 2 a 4 
de todos os exercícios. A exceção foi a rosca bíceps que apresentou diferenças nas séries 1 a 3 , sem diferença entre os grupos na série 4 .

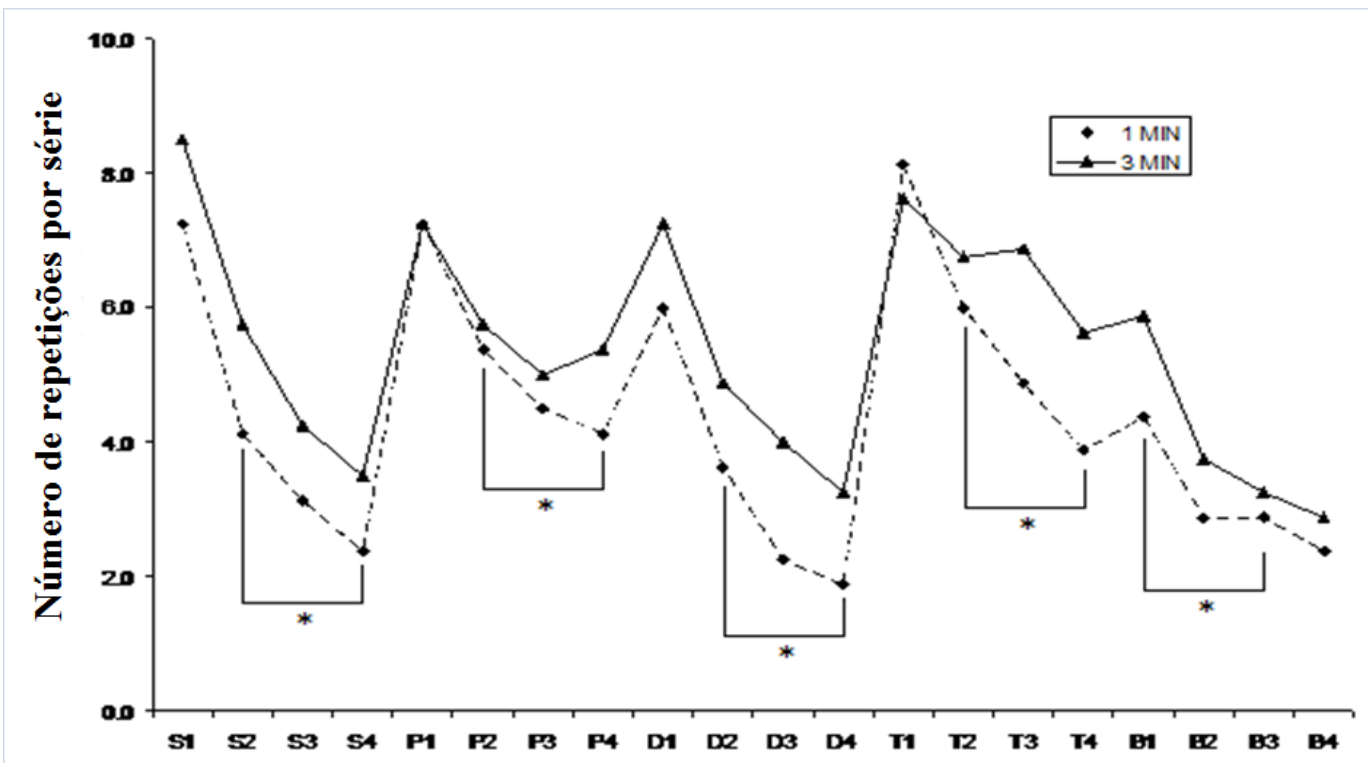

Gráfico 1 - Número de repetições por série em cada um dos exercícios para cada um dos intervalos. *representa diferenças significativas entre as sessões com 1 e 3 minutos de intervalo de descanso entre as séries ( $<<0,05)$.

Houve diferença significativa $(\mathrm{p}<0,05)$ entre o volume total de cada um dos exercícios realizados nas sessões com 1 e 3 minutos de intervalo (Tabela 3). Houve exceção para a puxada no pulley.

Tabela 3 - Volume total (séries $\mathrm{x}$ repetições $\mathrm{x}$ carga). (*) representa diferença significativa entre 1 e $3 \mathrm{~min}(\mathrm{p}<0,05)$.

\begin{tabular}{lccc}
\hline & $1 \mathrm{~min}$ & $3 \mathrm{~min}$ & $\mathrm{P}$ \\
\hline Supino Reto $(\mathrm{Kg})$ & $1196 \pm 369$ & $1567 \pm 502^{*}$ & 0,01 \\
Puxada no Pulley $(\mathrm{Kg})$ & $1710 \pm 367$ & $1876 \pm 311$ & 0,09 \\
Desenvolvimento $(\mathrm{Kg})$ & $793 \pm 192$ & $1134 \pm 441^{*}$ & 0,02 \\
Rosca Tríceps $(\mathrm{Kg})$ & $1706 \pm 299$ & $2025 \pm 615^{*}$ & 0,02 \\
Rosca Bíceps $(\mathrm{Kg})$ & $610 \pm 336$ & $764 \pm 374^{*}$ & 0,01 \\
\hline
\end{tabular}

Houve um aumento significativo da atividade sérica de CK nas medidas de 24, 48 e 72 horas em comparação com a medida PRE $(\mathrm{p}<0,05)$ como pode ser observado no gráfico 2. Não houve diferença significativa quando comparadas as sessões de exercícios com intervalo de descanso de 1 e 3 minutos. 
Gráfico 2 - Variação da atividade sérica de CK em ambos os tempos de intervalo entre as séries e exercícios. (*) representa diferença significativa em relação a PRE $(\mathrm{p}<0,05)$.

A atividade sérica de AST aumentou significativamente após a realização dos exercícios conforme pode ser observado no gráfico $3 \quad(\mathrm{p}<0,05)$. Não houve diferença significativa entre 1 e 3 minutos de intervalo de descanso.

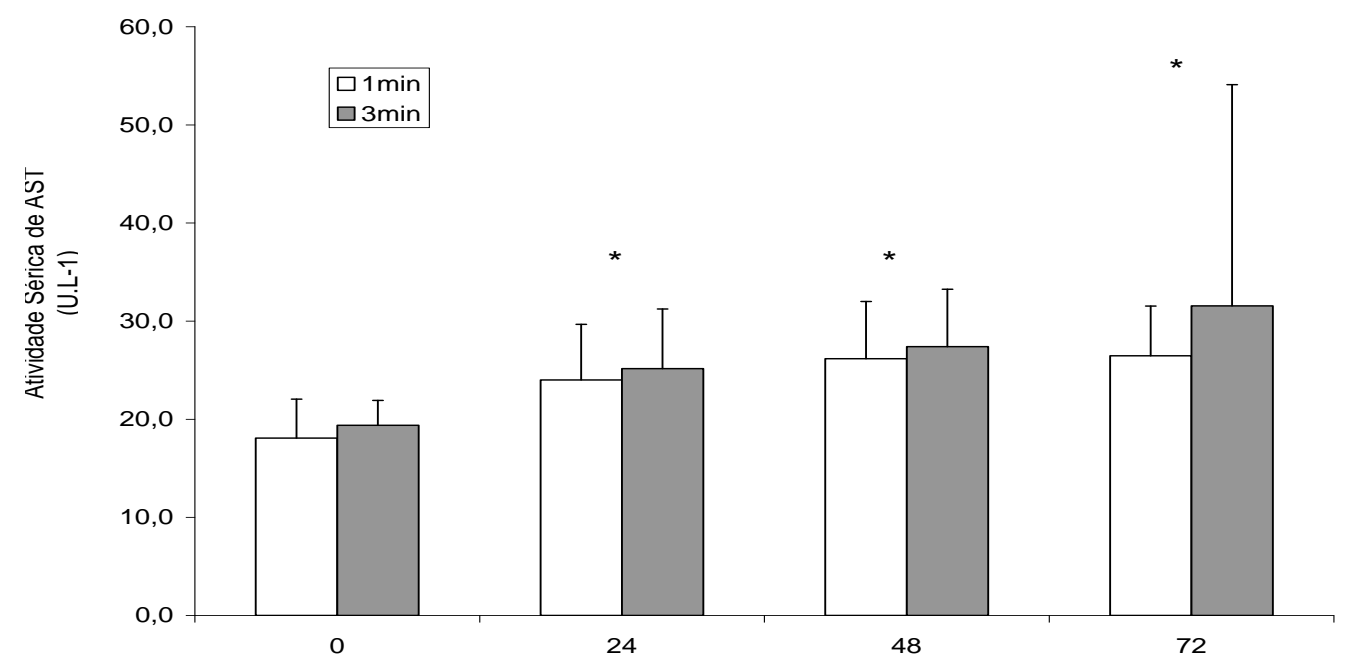

Gráfico 3 - Variação da atividade sérica de AST em ambos os tempos de intervalo entre as séries e exercícios. (*) representa diferença significativa em relação a PRE $(\mathrm{p}<0,05)$.

Não houve diferença significativa na atividade sérica de ALT em nenhum dos momentos e nem entre as sessões (Gráfico 4).

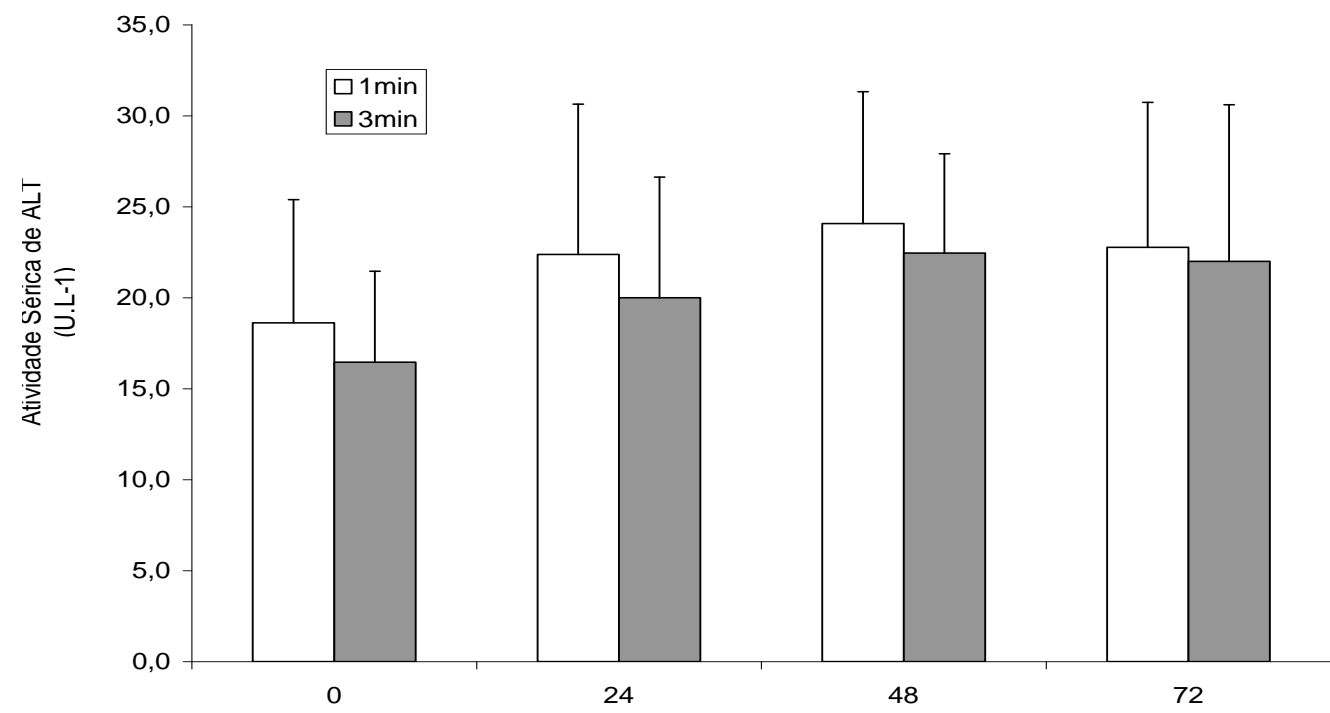

Gráfico 4 - Variação da atividade sérica de ALT em ambos os tempos de intervalo entre as séries e exercícios. (*) representa diferença significativa em relação a PRE $(\mathrm{p}<0,05)$. 


\section{4 - Discussão}

Corroborando estudos anteriores (LIMA et al., 2006; SALLES et al., 2006; SIMÃO et al., 2006a; SIMÃO et al., 2006b; SIMÃO et al., 2008; MIRZAEI et al., 2008; SENNA et al., 2009; RODRIGUES et al., 2010b) houve uma redução significativa no número de repetições executados a cada série. Além disso, a redução sempre foi maior nas repetições dos exercícios executados com intervalo de 1 minuto. A redução no número de repetições se explica pela instalação de mecanismos de fadiga (acidose, aumento de íons fosfato, desequilíbrio iônico na membrana, depleção de substratos) induzidos pelo exercício máximo (DUARTE et al., 2008) e pelo tempo reduzido para recuperação (WILLARDSON, 2006).

Houve uma diferença significativa no volume total, conforme pode ser observado na tabela III, achado que também corrobora os estudos supracitados. Estes dados corroboram especialmente o estudo de Rodrigues e colaboradores (2010b) em que somente exercícios para a parte superior do corpo foram incluídos na sessão. $\mathrm{O}$ volume de cada exercício, bem como o volume total de treino, foi significativamente menor na sessão com intervalos mais curtos.

A atividade sérica de $\mathrm{CK}$ aumentou significativamente 24 horas após as duas sessões de exercício, achados que confirmam os dados da literatura (TRICOLI, 2001; MOTA, 2003; PROSKE \& ALLEN, 2005; FOSCHINI et al., 2007; MACHADO, 2008). Esse aumento permaneceu até as $96 \mathrm{~h}$ após a sessão de exercícios, e isso é explicado pelo tempo para que a CK liberada pela musculatura alcance o sistema linfático, passe para o sangue e seja depurado pelo sistema reticulo endotelial. Motta (2003) relata que os valores de CK podem permanecer aumentados por até uma semana após o dano muscular.

De forma semelhante a atividade da CK, a atividade sérica de AST aumentou nas primeiras 24 horas após o exercício, permanecendo elevada durante as $96 \mathrm{~h}$ do experimento. Motta (2003) descreve que a AST pode permanecer elevada até 5 dias após danos musculares e infarto do miocárdio, corroborando os resultados do presente estudo. Ainda de forma semelhante aos achados da CK, não houve diferenças significativas entre as sessões de exercício com diferentes intervalos de descanso entre as séries.

Os achados de aumento das atividades de CK e AST sem diferenças entre as sessões confirma achados anteriores de Ribeiro et al. (2008), Rodrigues et al. (2010a), Machado et al. (2011) e Evangelista et al. (2011). Rodrigues e colaboradores (2010a), utilizando exclusivamente exercícios para a parte superior do tronco como no presente estudo, encontraram aumentos da atividade sérica de CK e de lactato desidrogenase (LDH) nas sessões com diferentes intervalos de descanso (1 e 3 minutos), sem que houvesse diferenças significativas na comparação entre as sessões. Resultado semelhante foi encontrado por Evangelista et al. (2011), utilizando apenas a rosca bíceps no banco Scott. Os mesmos resultados foram descritos por Ribeiro et al. (2008) utilizando uma sequência de exercícios que incluía membros superiores e inferiores.

Porém, em um estudo avaliando indivíduos habituados ao treinamento militar, Curty e Bara Filho (2011), não encontraram aumentos significativos na atividade sérica de CK nos intervalos de 24, 48 e 72 horas após o teste de aptidão física do tiro de guerra, realizando exercícios intermitentes alternando membros superiores e inferiores, mesmo com alta intensidade avaliada através da escala subjetiva do esforço proposta por Borg em 1982. No 
presente estudo não foram encontradas diferenças significativas na atividade sérica de ALT em nenhum momento do estudo. Estes resultados confirmam os dados de Motta (2003), que cita que essa enzima não possui especificidade para danos no tecido muscular, apesar de também ser encontrada nas fibras musculares.

\section{5 - Conclusões}

A partir dos achados do presente estudo pode-se concluir que (1) intervalos mais curtos de repouso entre as séries reduz o volume de repetições que o executante consegue realizar durante as séries, (2) que uma sessão de exercícios de força causa aumento na atividade sérica de CK e AST (3) sem que os diferentes intervalos entre as séries cause diferenças nestas variáveis bioquímicas.

\section{6 - Agradecimentos}

Ao Professor Marco Machado do Laboratório de Fisiologia e Biocinética da UNIG Campus V pelas sugestões.

\section{7 - Referências}

BARROSO, R.; TRICOLI, V.; UGRINOWITSCH, C. Adaptações neurais e morfológicas ao treinamento de força com ações excêntricas. Revista brasileira de ciência e movimento, v. 13, n. 2, p. 111-122, 2005.

CURTY, V. M.; BARA FILHO, M. G. Estado de recuperação avaliado através de dois métodos após teste de aptidão física. Brazilian Journal of Biomotricity. v. 5, n. 3, p. 186-199, 2011.

DUARTE, V. L.; DIAS, D. S.; MELO, H. C. S. Mecanismos moleculares da Fadiga. Brazilian Journal of Biomotricity, v. 2, n. 1, p. 3-38, 2008.

EVANGELISTA, R.; PEREIRA, R.; HACKNEY, A.C.; MACHADO, M. Rest interval between resistance exercise sets: length affects volume but not $\mathrm{CK}$ activity or muscle soreness. International Journal of Sports Physiology and Performance, v. 6, p. 118-127, 2011.

FOSCHINI, D.; PRESTES, J.; CHARRO, M. A.; Relação entre exercício físico, dano muscular e dor muscular de início tardio. Revista Brasileira de Cineantropometria e Desempenho Humano. v. 9, n. 1, p. 101-106, 2007.

LIMA, F. V.; CHAGAS, M. H.; CARRADI, E. F. F.; SILVA, G. F.; SOUZA, B. B.; MOREIRA JUNIOR, L. A. Análise de dois treinamentos com diferentes durações de pausa entre as séries em normativas previstas para hipertrofia muscular em indivíduos treinados. Revista Brasileira de Medicina do Esporte. v. 12, n. 4, p. 175-178, 2006

MACHADO, M. Atualidades em fisiologia do músculo esquelético - células satélites e hipertrofia. Perspectiva Online. v. 5, n. 1, p. 116-120, 2008. 
MACHADO, M. O papel dos micros-traumas e das células satélites na plasticidade muscular. Arquivos em movimento. v. 3, n. 1, p. 113-121, 2007.

MACHADO, M.; KOCH, AJ ; WILLARDSON, JM ; PEREIRA, LS ; CARDOSO, M.I.; MOTTA, MKS ; PEREIRA, R. ; MONTEIRO, A N. Effect of varying rest intervals between sets on creatine kinase and lactate dehydrogenase responses. Journal of Strength and Conditioning Research, v. 24, p. 1339-1345, 2011.

MACHADO, M.; WILLARDSON, JM. Short Recovery Augments the Magnitude of Muscle Damage in High Responders. Medicine and Science in Sports and Exercise, p. 1-1374, 2010.

MACHADO, M.; KOCH, AJ ; WILLARDSON, JM ; SANTOS, F C ; CURTY, V. M. ; PEREIRA, L. N. . Caffeine Does Not Augment Markers of Muscle Damage or Leukocytosis Following Resistance Exercise. International Journal of Sports Physiology and Performance,, v. 5, p. 18-26, 2010.

MAYHEW, DL; THYFAULT, JP; KOCH, AJ. Rest-interval length affects leukocyte levels during heavy resistance exercise. Journal of Strength and Conditioning Research. 19:16-22, 2005.

MIRZAEI, B.; NIA, F. R.; SABERI, Y. Comparison of 3 different rest intervals on sustainability of squat repetitions with heavy vs. light loads. Brazilian Journal of Biomotricity, v. 2, n. 4, p. 220-229, 2008

MOTTA, V. T. Bioquímica Clínica: Princípios e Interpretações. Porto Alegre: Missau, 2003.

PRESTES, J.; FOSCHINI, J.; MARCHETTI, P.; CHARRO, M. A. Prescrição e periodização do treinamento de força em academias. Barueri/Manole, 2010.

PROSKE, U.; ALLEN, T. J. Damage to Skeletal Muscle from Eccentric Exercise. Exercise and Sports Science Reviews, v. 33, n. 2, p. 98-104, 2005.

RIBEIRO, V. F. S.; PEREIRA, R.; MACHADO, M. Resistance exercise-induced microinjuries do not depend on 1 or 3 minutes rest time interval between series. International Journal of Sport Science. v. 5, n. 13, p. 44-53, 2008.

RODRIGUES, B. M.; DANTAS, E. H. M.; SALlES, B. F.; MIRANDA, H.; KOCH, A. J.; WILLARDSON, J. M.; SIMAO, R. Creatine Kinase and Lactate Dehydrogenase Responses After Upper-Body Resistance Exercise With Different Rest Intervals. Journal of Strength and Conditioning Research, v. 24, p. 1657-1662, 2010a.

RODRIGUES, B. M.; SANDY, D. D.; MAZINI FILHO, M. L.; SOUZA JÚNIOR, J. J.; VENTURINI, G. O. R.; DANTAS, E. H. M. Sessão de treinamento resistido para membro superior com dois diferentes tempos de intervalo: efeitos na percepção subjetiva de esforço. Brazilian Journal of Biomotricity, v. 4, n. 2, p. 131-139, 2010b. 
SALLES, B. L.; RIBEIRO, F. M.; SILVA, J. P. M. R.; NOVAES, J. S. A influência de dois minutos de recuperação entre as séries sobre o número de repetições máximas em exercícios mono e bi articulares. Arquivos em movimento. v. 2, n. 2, p. 64-69, 2006.

SENNA, G; SALLES, BF; PRESTES, J; MELLO, RA; SIMÃO, R. Influence of two different rest interval lengths in resistance training sessions for upper and lower body. Journal of Sports Science and Medicine, 8:197-202, 2009.

SILVA, DP; CURTY, V. M.; AREAS, JM; Souza, SC ; HACKNEY, AC; MACHADO, M. Comparison of DeLorme with Oxford resistance training techniques: effects of training on muscle damage markers. Biology of Sport, v. 27, p. 77-81, 2010.

SIMÃO, R.; MONTEIRO, W.; JACOMEO, A.; TESSEROLI, C.; TEIXEIRA, G. A influência de três diferentes intervalos de recuperação entre séries com cargas para 10 repetições máximas. Revista brasileira de ciência e movimento, v. 14, n. 3, p. 37-44, 2006 a.

SIMÃO, R.; POLITO, M.; MIRANDA, H.; CAMARGO, A; HOOLLER, H.; ELIAS, M.; MAIOR, A. S. Analise de diferentes intervalos entre as séries em um programa de treinamento de força. Fitness \& Performance Journal. v. 5, n. 5, p. 290-204, 2006b.

SIMÃO, R.; POLITO, M.; MONTEIRO, W. Efeito de diferentes intervalos de recuperação em um programa de treinamento de força para indivíduos treinados. Revista Brasileira de Medicina do Esporte. v. 14, n. 4, p. 353-356, 2008.

TRICOLI, V. Mecanismos envolvidos na etiologia da dor muscular tardia. Revista Brasileira Ciência e Movimento, v. 9, n. 2, p. 39-44, 2001.

WILLARDSON, JM. A Brief Review: Factors Affecting The Length Of The Rest Interval Between Resistance Exercise Sets. Journal of Strength and Conditioning Research 20:978-84, 2006. 